\title{
Buchbesprechungen - Book Reviews - Livres nouveaux
}

Journées annuelles de diabétologie de l'Hôtel-Dieu. M. Dérot ed. Flammarion. Paris. 1962. 207 p. Prix NF 42.-.

Die vom 10.- 12. Mai 1962 im wichtigsten Diabeteszentrum in Paris gehaltenen Vorträge geben eine gute Ubersicht über die zurzeit intensiv bearbeiteten Probleme der Zuckerkrankheit. Es handelt sich zum Teil um zusammenfassende Darstellun-gen, welche den Status quo unserer Kenntnisse und Vorstellungen klar und präzis vermitteln, zum Teil aber auch um Mitteilungen eigener, neuer Untersuchungs-befunde. Die Themen umfassen ein breites Feld: Neuere Ergebnisse der Insulin-forschung (Struktur, Bestimmungsmethoden, Wirkungsmechanismus, Insulin und Pathogenese des Diabetes) (Pignard), Insulinresistenz (Larcan), Funktionspriifung des Inselapparates (Dérot, Dousset, Pellegrini, Bernier), labiler Diabetes (Plauchu, Mirouze), Schilddrüse und Diabetes (Sendrail) Mucoviscidosis und Diabetes (Klotz), Komplikationen: Fettstoffwechsel und Atheromatose (Warembourg), Retinopathie und anabole Hormone (Tausk), Glomerulosklerose (Reubi, Thomsen, Sue), Haemo-chromatose (Rambert), Therapie: Heilung (?) des Diabetes beim Jugendlicben (Deuil, Laurent), Tolbutamid in der Pädiatrie (Lestradet), Wirkungen von oralen Antidiabetica auf Schwangerschaft und Entwicklung des Föt.us bei der Ratte (Tuchmann). Der hohe Prozentsatz von Aborten und Mißbildungen, insbesondere bei Carbutamid, gibt zum Denken Anlass.

Das Niveau der Journées de diabétologie ist überlegen, so daß auf Einzelheiten nicht eingegangen werden muß. Zwei Wünsche an den Herausgeber: 1. Abkürzungen sind für den fremdsprachigen Leser oft unverständlich. 2. In der Bibliographie ware die in seinem Lande verpönte Pedanterie anaiebracl·t. Kapp, Basel H.Schön: Fettstoffwechsel und Âtherosklerose. Sonderheft Medizin und Ernährung. 37 Seiten, 17 Abb., 8 Tabellen. DM 9.80. Pallas-Verlag, Lochham/München. 1962. Die kleine Monographie zerfällt in zwei Teile. In dem relativ kurzen ersten Teil (16 Seiten) ist ein Maximum von Information über die Beziehungen des Fettstoffwechsels zur Âtherosklerose in klarer und übersichtlicher Weise integriert. Fast 700 Literaturzitate bilden die Grundlage und zeigen die enorme und verdienstliche Ordnungsarbeit des Autors. Klinik, Formale Pathogenese, Endogene Faktoren, Cholesterinstoffwechsel, Exogene Faktoren, Atherogenese-Theorien, Experimentelle Modelle bilden die einzelnen konzis gefaßten Kapitel. Im zweiten Teil stellt der Autor eigene Untersuchungen zur Wirkungsweise der Polyensäuren dar. Diese umfaßt eine Steigerung der Cholesterinsynthese in der Leber und eine Steigerung der Ketogenese. Diese ist auf eine Beschleunigung der Klärung dieser Fette, bewirkt durch einen rascheren Umsatz der polyensäurehaltigen Chylomikronen-Triglyceride, zurückzuführen. Eine Klärinsuffizienz wurde bei Âtherosklerose und zu Âthero sklerose disponierenden Erkrankungen nachgewiesen. Die Lipoproteidlipase steht im Zentrum der Pathogenese. Neue Teste werden mitgeteilt. Kapp, Basel Fettsucht, Pathogenese, Klinik und Therapie. Sonderheft Medizin und Ernährung. 50 Seiten. DM 16.-. Pallas Verlag. Lochham/München, 1962. 
Das vorliegende Sonderheft enthält die Vorträge der von N. Henning, Erlangen, geleiteten Fachgruppe «Klinische Biologie der Fette», die anläßlich der Tagung der Deutschen Gesellschaft für Fettwissenschaft am 25./26.0ktober 1961 in Hamburg gehalten wurden.

76 Buchbesprechungen - Book Reviews - Livres nouveaux

Fettsucht ist für die Medizin ein bedeutsames und irgendwie verwirrendes Thema. Es wird daher immer wieder überprüft, ob die Grundlagenforschung Fort-schritte verzeichnet und, ob diese Fortschritte geeignet sind, eine befriedigendere Theorie der Fettsucht zu entwickeln.

Über Grundlagenforschung informieren eine Reihe aufschlussreicher Arbeiten: Eger (Göttingen), Struktur und Funktion des Fettgewebes; Ries (Leipzig), Chemie des Körperfettes; Leupold (Köln), Intermediärstoffwechsel bei Fettsucht: Frank (Erlangen), Wasser- und Salzhaushalt bei Fettsucht; Berg (Erlangen), Eiweiß- und Lipoidrelation der Serumfraktionen als Charakteristikum der Lipoproteide.

Bahner (Heidelberg) eròrtert grundsätzlich Bilanz- und Regulationsfragen. Bansi (Hamburg) gibt eine ausgezeichnete Übersicht über die heute wahrscheinlichste Konzeption der Pathogenese mit der Einteilung in regulativ und metabolisch ge-störte Energiebilanz. Boiler (Wien) berichtet in origineller Weise über die empirisch orientierte Diätetik der Fettsucht. Die Pharmakologie der appetitmindernden Substanzen wird von Loser und Opitz (Münster), die medikamentöse Therapie der Fettsucht von Schön (Erlangen) dargestellt.

Mit dieser Inhaltsangabe wird gezeigt, daß die kleine Monographie in vorzüglicher Weise über den Stand unserer Kenntnisse auf dem Gebiete der Fettsucht orientiert.

Kapp, Basel

Somogyi, J. C.: Emährungswissenschaft im Dienste der Entwicklungsländer. Bibliotheca «Nutritio et Dieta», Fasc. 4, S. Karger, edit. Bale (1962), 262 p., 27 figures, 64 tableaux, sFr. 47.-

Le dernier fascicule de la Bibliotheca Nutritio et Dieta est consacré à un problème de grande actualité: celui de $\Gamma$ alimentation dans les pays en voie de développement, auquel fut consacré un Symposium à Feldach et Zurich, en octobre 1961.

Les principaux rapports envisagèrent la couverture du besoin calorique (Gounelle, Paris), le deficit protéinique (Bígwood, Bruxelles), les apports lipidiques (Bernhard, Basel), vitaminiques (Kühnau, Hamburg) et calciques (Eeg-Larsen, Oslo). D'autres travaux étudièrent les problèmes soulevés par Talimentation des animaux (Schürch et Crasemann, Zurich), la fertilité du sol (Holmes, Ashford); et enfin on n'oublia pas les problèmes éducationnels (Yudkín, London), sociaux et économiques (Ferro-Luzzi, Rome).

Clairement exprimé, illustré de quelques schémas très évocateurs, ce volume retiendra Гattention de tous ceux que préoccupe ce qu'on a appelé, dans un affreux jeu de mots, la «faim du monde». M.Demole, Geneve Principes devant régir la protection de la santé des consommateurs à $\Gamma$ égard des résidus de pesticides: Rapport d'une reunion d'un Comité OMS d'experts des Résidus de Pesticides, tenue conjointement avec le Groupe d'experts FAO sur Гemploi des produits antiparasitaires en agriculture. Organisation mondiale de la Santé, Série de Rapports techniques, $\mathrm{n}^{\circ} 240 ; 21$ pages. Prix: sFr. 1.-, 1/9, \$ 0.30. Egalement publié en anglais et en espagnol. En vente chez Huber, Bern.

Selon ce rapport, quatre conditions essentielles doivent être remplies si 1'on veut écarter à coup sûr les dangers que peuvent presenter les résidus de pesticides. Les services officiels intéressés devront faire appel à des conseillers agricoles qualifies connaissant bien les habitudes des cultivateurs du pays et les principales cultures qu'ils pratiquent, aménager des laboratoires pour 
Tanalyse des résidus, obtenir auprès d'experts en toxicologie des avis sur les quantités de pesticides qui peuvent être ingérées quotidiennement sans dommage et organiser des consultations entre

Buchbesprechungen - Book Reviews - Livres nouveaux 77

toutes les administrations intéressées. Le rapport expose divers moyens de remédier aux consequences préjudiciables de Гemploi des pesticides; il met $\Gamma$ accent sur la nécessité d'enregistrer ces produits et de prévoir des étiquettesportant les indications voulues. II définit la dose journalicre acceptable pour une absorption prolongée, la concentration autorisable et la tolerance, et explique comment calculer ces diffé-rentes grandeurs.

Le rapport recommande d'intensifier les recherches sur le métabolisme des in-vertébrés, des plantes et des animaux à sang chaud en vue de mettre au point de nouveaux insecticides ayant une toxicité elective à $\Gamma$ égard de parasites particuliers tout en étant inoffensifs pour Thomme. II souligne en outre Гintérêt de créer un service de documentation scientifique et legislative qui aurait pour tâche de re-cueillir, classer et diffuser des renseignements publiés ou inédits sur tout ce qui a trait aux pesticides utilises en agriculture et dans les entrepots de produits aîmen-taires. Charles Alaís: Science du lait et principe des techniques laitières. Edition Sepaic, Paris, 450 pages, NF 34.-.

Cet ouvrage présente un ensemble de notions fondamentales concernant le lait et les produits laitiers. II s'agit surtout de données scientifiques nouvelles ou confirmees et de principes dont la connaissance est indispensable à tous ceux qui s'intéressent à la production du lait, à ses transformations, à $\Gamma$ hygiène du lait et aux produits laitiers considérés comme des aliments essentiels.

L'auteur est à la fois charge d'enseignement à YInstitut Agrícole de Beauvais et chercheur à la Station Centrale de Mícrobíologie et Recherches laitières de Jouy-en-Josas (I.N.R.A.). II a bénéficië de l'importante documentation réunie dans cet Institut et de $\Gamma$ appui de ses collègues chimistes, microbiologistes, biochimistes, nutri-tionnistes, vétêrinaires et zootechniciens. II a aussi tire profit de sa propre experience dans le domaine complexe des matières azotées du lait. Dans Vintroduction sont passées en revue les propriétées fondamentales du lait et les caractéristiques principales des laits de différentes espèces.

La premiere partíe traite de la constitution du lait, des propriétés des composants classes en glucides, lipides et substances associées, protides, minéraux, enzymes et vitamines. Les propriétés physiques du lait sont étudiêes ainsi que les modifications apportées par divers traitements, notamment le chauffage.

La microbiologie et les problèmes hygiéniques sont exposes dans la deuxième partíe:

contamination banale, flore lactique, bactéries pathogènes, destruction des germes par la chaleur, consequence de $\Gamma$ infection de la mamelle.

La troísième partíe est consacrée à la production du lait et la quatríème partíe aux traitements et transformations du lait.

Les médecins et diététiciens trouveront dans cet ouvrage, qui n'a pas d'équi-valent en langue française, des renseignements utiles à $\Gamma$ exercice de leur spécialité.

J. Trémolíères

Henri de Farcy: Produire et vendre. Editions Spes, Paris.

Le livre de H. de Farcy doit être lu et médité par tous ceux qui s'intéressent à la production, à

l'information, à $\Gamma$ instruction, à la commercialisation des denrées alimentaires.

Au moment où les structures paysannes françaises sont ébranlées par le dévelop-pement industriel, par les transformations d'un marché élargi, par le progrès technique, par les pressions 
financières Internationales, il est fondamental que chacun réfléchisse profondémeut sur le sens de ces transformations.

78 Buchbesprechungen - Book Reviews - Livres nouveaux

Le livre de H. de Facy en fournit la matière. Sans these à priori, suggérant plus qu'il ne résout, il présente une situation. II suggère les problèmes auxquels c'est à chacun de réfléchir. Les nombreux échecs et les quelques succès d'extension de marches alimentaires qu'il rapporte, montrent bien que dans un pays dont la «culture» est si intimement liée à $\Gamma$ «agriculture», dont la vie sociale s'exprime tant autour de la table, où le repas familial, le repas d'affaires, les banquets politiques, le repas en tête-à-tête, sont de tels moyens de communication, Гaliment constitue une symbolique si précieuse que ce n'est pas un simple objet de marché, mais une marque de savoirvivre.

Puisse ce travail contribuer à démarrer et à développer une politique alimentaire

saine et humaine. J. Trêmolíères, Paris

H. Braunsteíner, S. Sailer und F. Sandhofer: Lipoproteidlipase. Bibliotheca Haemato-logia, Fasc. 15, Symposium vom 1. und 2.Juni 1962 an der 2. Medizinischen Universitätsklinik, Wien.

S.Karger AG, Basel 1963, IV + 148 S., 58 Abb.,

4 Tab. Preis: sFr./DM 27.-.

Der wichtige Fragenkomplex der Lipoproteidlipase wurde in einem Symposium bearbeitet, wobei vor allem auf methodische Fragen näher eingegangen wurde. Dies ist besonders dankenswert, da im derzeitigen Stadium weitere Untersuchungen dringend notwendig sind und auf möglichst sicheren methodischen Voraussetzungen beruhen müssen. Es kann nicht auf alle Beiträge im einzelnen eingegangen werden, es sei nur auf das Einleitungsreferat von O.Kraupp (Wien), auf den Bericht von H. Braunsteíner (Wien) über seine eigenen Arbeiten auf diesem Gebiet sowie auf den kritischen Beitrag N.Zöllners (München) über den Wirkungsmechanismus von Heparin im Fettstoffwechsel hingewiesen. Weitere Beiträge, unter anderem von

B.Kommerell (Berlin) und M.Eggsteín (Marburg an der Lahn) befaßten sich mit Fragen der Wechselbeziehungen zwischen Fetten und Blutgerinnung.

Im klinischen Teil referierte vor allem G. Schettler (Berlin) über die Rolle der Lipoproteidlipase im Arteriosklerosegeschehen.

Der Band gibt einen guten Überblick über den derzeitigen Stand unseres Wissens um das Ferment Lipoproteidlipase. Die darin behandelten Fragen sind heute nicht nur für den Theoretiker, sondern auch für den Kliniker wichtig.

R. Wenger, Wien

Protein Metabolism. Influence of Growth Hormone, Anabolic Steroids and Nutrition in Health and Disease. An International Ciba Symposium. Leyden, 25th-29th June, 1962. Sponsored by Ciba, Chairman A. Querido, Edited by F. Gross. Springer-Verlag, Berlin-Göttingen-Heidelberg, 1962, XII + 521 Seiten, 159 Abbildungen, $8^{\circ}$. Preis: Ganzleinen DM 37.50.

Rapports et discussions d'un Symposium Ciba groupant l'état actuel des con-naissances sur le métabolisme des protéines sous 5 chapitres:

- Uaction des hormones au níveau cellulaíre présente les effets de Thormone de croissance, des androgènes et des æstrogènes sur la synthèse protéique étudiée à Гaide de microsomes isolés et d'amino acides marques d'une part, par le microscope électronique d'autre part. L'hormone de croissance active le pouvoir de fractions microsomiques à synthétiser des protéines; cet effet serait dû à un effet sur le micro-some et non pas sur les systèmes activant les amino acides. Les androgènes et les æstrogènes activent la synthèse protéique en activant la transformation des complexes ARN-acides amines solubles en ribonuclêoprotéines microsomiques. Ces deux 
groupes d'hormones agissent de façon assez identique sur la cellule dont ils dévelop-pent le microsome, les mitochondries et grains de secretion. Leur effet global ana-

Buchbesprechungen - Book Reviews - Livres nouveaux 79

bolisant ou catabolisant depend des tissus sur lesquels ces hormones ont agi et de l'équilibre endocrinien et nutritionne general.

Les facteurs ínfluant sur le métabolísme protéique à Vechelon de Vorganisme comportent d'abord le taux protéique des ingestations réglant la labilité des diverses protéines tissulaires. Ces regulations se font de façon différente chez 1'enfant et Tadulte, expliquant les différents types de croissance suivant l'âge où $\Gamma$ on modifie le niveau alimentaire.

Les facteurs nutritionnels jouent avec les facteurs endocriniens sans qu'on puisse étudier les uns sans les autres. Ils agissent simultanément au niveau des cellules, de la repartition de la masse protéique entre les divers tissus et au niveau du mésencé-phale.

Les méthodes d'études et le mode d'actíon des stéroîdes anabolisants comportent les effets sur les poids d'organes spécifiques. Les effets different suivant l'état endocrinien, suivant le taux et la valeur biologique des protéines du regime.

Les modifications du métabolísme protéique en pathologie sont étudiées au cours de la maladie opératoire, des cirrhoses.

Ce livre constitue la mise au point de ces sujets la plus approfondie à ce jour.

Olsen, E.S. et Quaade, F.: Fatty foods and obesity. Lancet; 1: 1048-1051 (mai 1960.) Ce très intéressant article semble mettre fin aux theories de Pennington et de Kekwick et Pawan. Les auteurs se sont attaches à élucider cette anomalie de la saine logique, dans des conditions de travail parfaitement rigoureuses, sur des obèses hospitalises, tous volontaires. L'étude a porté sur trois périodes, en 15 jours:

une premiere période de 7 à 10 jours de regime libre;

une deuxième période de regime hyperlipidique fournissant 2600 calories et ne contenant que 40 g d'hydrates de carbone;

une troisième période de regime hypocalorique (1,250 cal. en moyenne) et divisée en deux temps: le premier temps comprenait, pour 1000 cal. $32 \%$ de protéines, $50 \%$ de graisses et $18 \%$ de sucres; le deuxième: $32 \%$ de protéines, $18 \%$ de graisses et $50 \%$ d'hydrates de carbone.

Les conclusions des auteurs sont parfaitement nettes:

$1^{\circ}$ Un regime riche en graisses et pauvre en sucre peut faire perdre à Гobèse un bon nombre de kilos, mais cela uniquement par une perte d'eau; celle-ci cesse après quelques jours, sans que la reserve graisseuse de Tobèse soit entamée.

$2^{\circ} \mathrm{Si}$ Гon continue ce regime, le poids corporel varie comme il est prévu par la ration calorique totale administrée.

$3^{\circ} \mathrm{Si}$ Гon prolonge $\Gamma$ expérience sur plus de 3 semaines, l'amaigrissement obtenu est identique quel que soit le regime suivi, et strictement proportionnel à la privation calorique globale.

II semble done que les observations de Pennington soient surtout dues aux

fluctuations de l'hydratation corporelle, qui, en definitive, ne représentent qu'une

faible fraction du poids excédentaire. M.Dèmole

Techniques for Measuring Body Composition. Proceedings of a Conference. Quartermaster

Research and Engineering Center, Natick, Mass., January 22-23, 1959. Sponsored by

Headquarters Quartermaster Research and Engineering Command, U.S. Army Quartermaster

Corps, and Committee on Stress Physiology, Advisory Board on Quartermaster Research and

Development Division of Engineering and Industrial Research. Edited by Joseph Brozek, Lehigh 
University, and Austin Henschel, Quartermaster Research and Engineering Command. National Academy of Sciences - National Research Council, Washington D.C. 1961. 300 S.

80 Buchbesprechungen - Book Reviews - Livres nouveaux

In den letzten zwanzig Jahren ist man mehr und mehr dazu übergegangen, beim biologischen Vergleich von Menschen verschiedener Statur die ehrwürdige Gewicht/ Größe-Relation zu verlassen und präzisere Bezugsgrößen zu suchen. Die üblichen anthropologischen Methoden, die im wesentlichen zur Beschreibung der Körper-form dienen, schienen wenig geeignet, physiologische Vergleichsbasen zu liefern. Statt dessen wurde auf verschiedenen Wegen versucht, Indices für die stoffliche Zusammensetzung des Körpers, beziehungsweise die Größe der metabolisch wich-tigen Gewebe zu finden, wobei das wachsende Interesse an Problemen der Fett-sucht zweifellos eine der treibenden Kräfte war. Das vorliegende Buch stellt die Verhandlungen eines Symposiums im Januar 1959 dar und gibt einen Uberblick über den heutigen Stand dieser Bemühungen. In vier Kapiteln, «technical sessions », werden von 16 nordamerikanischen Autoren somatometrische, volumetrische und biochemische Methoden zur Messung der Körperzusammensetzung am Lebenden abgehandelt und miteinander verglichen. Jedes Kapitel beginnt mit einem einlei-tenden und in das Gebiet einführenden Aufsatz, dem mehrere Abhandlungen über die Anwendung spezieller Techniken folgen. Das erste Kapitel enthält ein ausführ-liches Übersichtsreferat über die verschiedenen indirekten Methoden zur Bestim-mung der Körperzusammensetzung einschließlich Anthropometrie. Dem folgen Arbeiten über die Anwendung von Röntgenaufnahmen zur Messung von subkuta-nem Fett, Muskelentwicklung und Knochendichte sowie eine Arbeit über die Bedeutung der Körperoberfläche als Bezugsgröße. Das nächste Kapitel ist der Messung der Körperdichte durch Unterwasserwägung nach dem archimedischen Prinzip und durch Messung der Verdünnung eines bekannten Gasvolumens, das einer die Versuchsperson enthaltenden Kammer beigemischt wird, gewidmet. Die Anwendung der Bestimmung von Körperwasser und Elektrolytverteilung wird im dritten Kapitel diskutiert, das außerdem eine Arbeit über Anderungen der Körperzusammensetzung bei experimenteller kalorischer Unterernährung enthält. Im vierten Kapitel werden die verschiedenen Methoden verglichen und korreliert und ihre Anwendung zur Gewinnung biologischer Standards diskutiert. Dem folgen ab-schließend Uberlegungen allgemeiner Natur zum Thema und Empfehlungen für weitere Forschung sowie ein gut redigierter Index. - Wie bei einem Buch, das aus einem Symposium entstanden ist, nicht anders zu erwarten, sind die einzelnen Methoden in unterschiedlicher Ausführlichkeit behandelt. Autoren außerhalb der USA kommen nicht zu Wort. Dies wird in hohem Grade wettgemacht durch die je-dem Artikel folgenden umfangreichen Literaturangaben. Gewisse Überschneidungen, besonders bei den densiometrischen Methoden, tragen eher dazu bei, das Gesamtbild zu beleben. Die durchweg klare Darstellung läßt nichts zu wünschen übrig. Als Ganzes stellt das Buch eine ausgezeichnete Übersicht dar über den heutigen Stand und frühere Arbeiten auf einem Arbeitsgebiet, das in Deutschland bisher wenige Anhänger hat, dem aber, nach der steigenden Zahl von Veröffent-lichungen zu schließen, in Zukunft mehr und mehr Bedeutung zukommen dürfte.

W.Keller 